\title{
Influencing Factors for Acceptance of Digital Tools in the Humanities
}

\author{
Einflussfaktoren für die Akzeptanz von digitalen Werkzeugen in den Geisteswissenschaften
}

\author{
Tobias Simon \\ Mainz University of Applied Sciences \\ Germany \\ tobias.simon@hs-mainz.de
}

\author{
Sven Pagel \\ Mainz University of Applied Sciences \\ Germany \\ sven.pagel@hs-mainz.de
}

\author{
Harald F.O. von Korflesch \\ University of Koblenz-Landau \\ Germany \\ harald.vonkorflesch@uni-koblenz.de
}

\section{ZUSAMMENFASSUNG}

English - Within the framework of the User Centered Design process, UX research is constantly exploring new fields of application and user groups. Especially in professional contexts, the question arises why certain user groups find it particularly difficult to integrate digital tools into their everyday work. The present study addresses this question by analysing the acceptance faktors, based on the Technology Acceptance Model, for the target group of often digitally inactive humanities scholars. In the humanities, digital tools are used to support research work, but the acceptance of using digital tools is relatively low or falls short of expectations despite the wide range of tools available. Therefore, the question arises what causes the lack of user acceptance and how this can be counteracted. This question is located at the intersection of the two disciplines Business Information Systems (BIS) and Human Computer Interaction (HCI). The present study contributes to answering this question by empirically determining the factors that influence the acceptance of use. In the present study, humanities scholars were familiarized with several digital tools in workshops and subsequently interviewed about them. Based on the collected data a model for usage acceptance was developed using the PLSSEM procedure. The most important factors for this target group are the perceived usefulness, which can be influenced by the social environment and the output quality of the tool, as well as the perceived ease of use, which can be influenced by positive user experiences.

Deutsch - Im Rahmen des User Centered Design Prozesses setzt sich die UX-Forschung immer wieder mit neuen Anwendungsfeldern und Nutzergruppen auseinander. Gerade im beruflichen Kontext stellt man sich in der Praxis oft die Frage warum es bestimmten Nutzergruppen besonders schwer fällt digitale Werkzeuge in ihren Arbeitsalltag zu integrieren? Dieser Frage widmet sich die vorliegende Arbeit, indem sie eine Akzeptanzfaktorenanalyse, in Anlehnung an das Technology Accepance Model, für die Zielgruppe der häufig noch wenig digital arbeitenden GeisteswissenschaftlerInnen, durchführt. In den Geisteswissenschaften werden zwar digitale Werkzeuge zur Unterstützung der Forschungsarbeit eingesetzt, jedoch ist

Permission to make digital or hard copies of part or all of this work for personal or classroom use is granted without fee provided that copies are not made or distributed for profit or commercial advantage and that copies bear this notice and the full citation on the first page. Copyrights for third-party components of this work must be honored For all other uses, contact the owner/author(s).

MuC'20, September 6-9, 2020, Magdeburg, Germany

(c) 2020 Copyright held by the owner/author(s).

ACM ISBN 978-1-4503-7540-5/20/09.

https://doi.org/10.1145/3404983.3405524 die Nutzungsakzeptanz digitaler Werkzeuge, trotz des mittlerweile umfangreichen Angebots, relativ gering beziehungsweise bleibt hinter den Erwartungen zurück. Es stellt sich demnach die Frage nach den Ursachen für die fehlende Nutzungsakzeptanz und wie dieser entgegengewirkt werden kann. Diese Fragestellung fällt in die Schnittmenge der beiden Fachdisziplinen Wirtschaftsinformatik (WI) und Human Computer Interaction (HCI). Die vorliegende Arbeit liefert einen Beitrag zur Beantwortung dieser Frage, indem sie die Einflussfaktoren der Nutzungsakzeptanz empirisch ermittelt. Im Rahmen der vorliegenden Studie wurden GeisteswissenschaftlerInnen im Rahmen von Workshops mit mehreren digitalen Werkzeugen vertraut gemacht und im Anschluss hierzu befragt. Aus den so erhobenen Daten wurde mit Hilfe des PLS-SEM-Verfahrens ein Modell für die Nutzungsakzeptanz entwickelt. Als wichtigste Faktoren stellen sich für diese Zielgruppe die wahrgenommene Nützlichkeit, beeinflussbar durch das soziale Umfeld und die Output Qualität des Werkzeugs, sowie die wahrgenommene Einfachheit der Bedienung, beeinflussbar durch positive Nutzungserfahrungen, heraus.

\section{CCS CONCEPTS}

- Software and its engineering $\rightarrow$ Software usability; Requirements analysis; Acceptance testing; Empirical software validation; • Human-centered computing $\rightarrow$ User studies; Field studies; HCI theory, concepts and models; Empirical studies in $\mathrm{HCI}$.

\section{KEYWORDS}

TAM, HCI, Technology Acceptance, DH, Digital Humanites, Akzeptanzfaktoren, Digitale Werkzeuge

\section{ACM Reference Format:}

Tobias Simon, Sven Pagel, and Harald F.O. von Korflesch. 2020. Influencing Factors for Acceptance of Digital Tools in the Humanities: Einflussfaktoren für die Akzeptanz von digitalen Werkzeugen in den Geisteswissenschaften. In Mensch und Computer 2020 (MuC'20), September 6-9, 2020, Magdeburg, Germany. ACM, New York, NY, USA, 11 pages. https://doi.org/10.1145/ 3404983.3405524

\section{EINLEITUNG}

Unter dem Sammelbegriff Geisteswissenschaften können aktuell über 40 einzelne wissenschaftliche Disziplinen zusammengefasst werden (vgl. Dizisplinen des Lexikon der Geisteswissenschaften [55]). Eine häufig verwendete Kategorisierung nach der Wissenschaftsgliederung von Anzenbacher sind die sechs Kategorien (1) Geschichtswissenschaften, (2) Kunstgeschichte, (3) Musikwissenschaften, (4) Literaturwissenschaften, (5) Religionswissenschaften und 
(6) Sprachwissenschaften [2]. Dieses Verständnis des Begriffs Geisteswissenschaften wird der vorliegenden Arbeit zu Grunde gelegt.

In den Geisteswissenschaften werden digitale Werkzeuge zur Unterstützung der Forschungsarbeit eingesetzt [29]. Als digitale Werkzeuge werden in der vorliegenden Arbeit Softwareanwendungen verstanden, welche die Forschenden im Forschungsprozess unterstützen. Diese Unterstützung erfolgt auf den drei Ebenen (1) Making, (2) Exploring und (3) Thinking [10]. Nach Harms und Grabowski können wissenschaftlich genutzte Softwaretools weiter anhand ihrer Spezialisierung in die drei Gruppen (1) General Software, (2) Generic Research Software und (3) Specific Research Software untergliedert werden [35]. In die erste Gruppe fallen Softwaretools, welche sich nicht alleinig der Forschung widmen, sondern vielmehr allgemeine Funktionen bieten, die auch außerhalb des Forschungskontextes eingesetzt werden [35]. Hierunter fallen beispielsweise Büroanwendungen, E-Mailprogramme, Betriebssysteme oder Messenger.

In die beiden weiteren Gruppen fallen Anwendungen, die speziell für die Forschung entwickelt und eingesetzt werden. Diese können entweder generischer Art sein, sodass sie in unterschiedlichen Forschungskontexten Anwendung finden. In diesem Fall werden sie der zweiten Gruppe zugeordnet. Als Beispiel seien hier Statistikoder Datenverwaltungsprogramme genannt. Oder sie sind spezifisch auf einen bestimmten Forschungskontext zugeschnitten. In diesem Fall werden sie der dritten Gruppe zugeordnet. Ein Beispiel für diese Gruppe aus dem Bereich der Geisteswissenschaften wäre der MEI Score Editor (MEISE) ${ }^{1}$, mit dessen Hilfe speziell kodierte Musikdokumente angezeigt und bearbeitet werden können.

Im Fokus der vorliegenden Arbeit stehen die beiden letzten Gruppen. Die Begriffe digitale Werkzeuge und Softwaretools werden im weiteren Verlauf der Arbeit synonym verwendet und entsprechend den obigen Ausführungen ausgelegt. Der Einsatz von digitalen Werkzeugen in den Geisteswissenschaften wird im Allgemeinen unter dem Begriff „Digital Humanities“(DH) zusammengefasst [66]. Golumbia definiert Digital Humanities als „anything that combines digital work of any sort with humanities work of any sort" [30].

1966 wird bereits der Einsatz von digitalen Werkzeugen im Journal Computers and the Humanities thematisiert. Ein Jahr später erscheint die erste Buchreihe zu diesem Thema (Computers in $\mathrm{Hu}$ manistic Research, [8]). Mittlerweile wird im Bereich der Geisteswissenschaften der Aufbau von digitalen Infrastrukturen auf nationaler und internationaler Ebene gefördert [13]. Beispielhaft seien hier die beiden nationalen Projekte Digital Research Infrastructure for the Arts and Humanities (DARIAH-DE) sowie Common Language Resources and Technology Infrastructure (CLARIN-D) genannt. Auf europäischer Ebene sind beide Projekte Teil des European Research Infrastructure Consortium (ERIC). Neben diesen Förderprojekten wurden in Deutschland in den vergangenen Jahren auch mehrere Kompetenzzentren für digitale Geisteswissenschaften, wie das „Trier Center for Digital Humanities“, das „Cologne Center for eHumanities“ oder das „Göttingen Centre for Digital Humanities“ aufgebaut und gefördert [12]. Ein weiteres Merkmal der fortgeschrittenen Digitalisierung auf diesem Feld ist die kaum mehr überschaubare Menge an digitalen Werkzeugen [11]. Einen guten Überblick über die Vielzahl der digitalen Werkzeuge für GeisteswissenschaftlerInnen

\footnotetext{
${ }^{1}$ https://de.dariah.eu/mei-score-editor
}

bieten diverse umfangreiche Sammlungen wie bspw. das „Digital Research Tools Directory“ 2 , die „Digital Toychest for Humanities“ 3 oder das „Text Analysis Portal for Research“ 4.

Wie im Folgenden dargelegt, ist die Nutzungsakzeptanz digitaler Werkzeuge, trotz des mittlerweile umfangreichen Angebots, relativ gering beziehungsweise bleibt hinter den Erwartungen zurück. So weist Borgmann darauf hin, dass die Nutzung von digitalen Werkzeugen in den Geisteswissenschaften längst nicht mit ähnlicher Selbstverständlichkeit stattfindet wie z.B. in den Naturwissenschaften [7]. Und das hat schon eine gewisse Tradition, denn bereits in den 1990er Jahren konnte eine mangelnde Nutzungsakzeptanz von digitalen Werkzeugen in den Geisteswissenschaften beobachtet werden. So argumentiert Warwick, dass in den 90er und frühen 2000er Jahren, die Anzahl der Journalartikel, welche eine geringe Nutzung digitaler Werkzeuge durch GeisteswissenschaftlerInnen beklagen, die Anzahl der Artikel, die über eine erfolgreiche Adaption von Softwaretools und Techniken berichten, deutlich übersteigen [76]. In einem jüngeren Artikel greift die Autorin diese Argumentation wieder auf und merkt an, dass obwohl seit dieser Zeit eine Menge Geld investiert wurde um digitale Werkzeuge und Methoden für Geisteswissenschaftliche Forschung publik zu machen, „Yet, the rate of change remained stubbornly slow." [77]. Auch der Abschlussbericht des „Summit on Digital Tools for Humanities“ zeichnet ein ähnliches Bild. Hier heißt es: „The broad availability of digital tools is a major development that has grown over the last several decades, but the use of digital tools in the humanities is, for the most part, still in its infancy [...] Only about six percent of humanist scholars go beyond general purpose information technology and use digital resources and more complex digital tools in their scholarship." [65].

Wie diese Ausführungen bereits andeuten und wie in Abschnitt 2 noch ausführlich dargestellt werden wird, wäre es zu einfach fehlendes Interesse oder Technologiefeindlichkeit von GeisteswissenschaftlerInnen für die geringen Nutzungszahlen verantwortlich zu machen. Es stellt sich demnach die Frage nach den Ursachen für die fehlende Nutzungsakzeptanz und wie dieser entgegengewirkt werden kann. Diese Fragestellung fällt in die Schnittmenge der beiden Fachdisziplinen Wirtschaftsinformatik (WI) und Human Computer Interaction (HCI). Die vorliegende Arbeit liefert einen Beitrag zur Beantwortung dieser Frage, indem sie die Einflussfaktoren der Nutzungsakzeptanz empirisch ermittelt. Diese Einflussfaktoren bilden die Grundlage für mögliche nachfolgende Forschungsarbeiten. So könnten Beispielsweise im Rahmen der Design Theorie Forschung auf Basis dieser Faktoren Maßnahmen zur Steigerung der Nutzerakzeptanz entwickelt werden. Dieser Folgeschritt ist jedoch nichtmehr bestandteil dieses Beitrags.

\footnotetext{
$\overline{{ }^{2} \text { https://dirtdirectory.org }}$

${ }^{3} \mathrm{http}: / /$ dhresourcesforprojectbuilding.pbworks.com

${ }^{4}$ http://tapor.ca
} 


\section{THEORETISCHER HINTERGRUND UND STAND DER FORSCHUNG}

\subsection{Einordnung des Themenfelds Technologieakzeptanzforschung}

Für die Untersuchung der Akzeptanz von neuen innovativen Technologien liefert im Speziellen die Akzeptanzforschung den benötigten theoretischen Hintergrund [18]. Die Technologieakzeptanzforschung beschäftigt sich im Kern mit der nutzerseitigen Betrachtung von neuen Technologien und versucht Annahme und Ablehnung dieser zu erklären. Im Laufe der Zeit wurden zu diesem Zweck unterschiedliche Modelle und Theorien entwickelt, die zur Erklärung der Nutzerakzeptanz unterschiedliche Einflussfaktoren heranziehen [67]. Die Technologieakzeptanzforschung greift dabei sowohl auf Konzepte der WI als auch auf Konzepte aus dem Bereich HCI zurück [82].

In der Literatur finden sich eine Vielzahl verschiedener Modelle zur Durchdringung und Akzeptanz von Innovationen wie die „Innovation Diffusion Theory - IDT“ [62], der „Task Technology Fit - TTF“ [32], das „Technology Acceptance Model - TAM“ [16], der „Combined TAM and TPB - C-TAM-TPB” [68] sowie der „Unified Theory of Acceptance and Use of Technology - UTAUT“ [74]. Deren Basis bilden etablierte Theorien aus der Verhaltensforschung wie die „Theory of Reasoned Action - TRA” [26] und die davon abgeleitete „Theory of Planned Behavior - TPB“ [1].

Im Folgenden wird das in der vorliegenden Arbeit zur Anwendung kommende „Technology Acceptance Model“ (TAM) kurz erläutert. Das Modell geht auf die Dissertation von Davis [16] zurück und wurde drei Jahre später von Davis et al. [19] zusammen mit einer Validierungsstudie [17] publiziert. Nach Angaben des Social Science Citation Index wurden die beiden Publikationen zusammen bereits fast 20.000 mal zitiert. Daher verwundert es auch nicht, dass das Technology Acceptance Model (TAM) in den letzten 3 Jahren mehrfach abgewandelt und erweitert wurde [46]. In diesem relativ simplen, auf der TRA [26] basierenden Modell, wird die Absicht eine Software zu nutzen (Behavioral Intention) von den beiden Determinanten Perceived Usefulness und Perceived Ease of Use bestimmt [17]. Wobei Davis Behavioral Intention in Anlehnung an die TPB von Ajzen zur Vorhersage des Nutzungsverhaltens (Use Behavior) einsetzt [1]. Die beiden zentralen Erweiterungen des ursprünglichen TAM bilden die Versionen TAM2 [73] und TAM3 [72]. Hierbei wurden im TAM2 das Konstrukt Perceived Usefulness und im TAM3 das Konstrukt Perceived Ease of Use weiter in Unterkonstrukte operationalisiert.

\subsection{Forschungsstand und Forschungsbedarf}

Das Problem der fehlenden Nutzungsakzeptanz wurde in Abschnitt 1 bereits angerissen. Hieran anknüpfend wird im Folgenden der Stand der Forschung zur Nutzungsakzeptanz von digitalen Werkzeugen skizziert. Im Bereich DH gibt es zu diesem Thema auch empirische Studien, welche die fehlende Nutzerakzeptanz bestätigen. Bei ihrer Befragung von 213 HistorikerInnnen stellen Gibbs und Owens beispielsweise fest, dass „Despite significant investment in digital humanities tool development, most tools have remained a fringe element in humanities scholarship." [28]. Zu einem ähnlichen Ergebniss kommen auch Toms und O’Brien. Sie stellen bei ihrer
Befragung von 169 GeisteswissenschaftlerInnen zum Thema digitale Textwerkzeuge fest, dass viele der Befragten die meisten, der gängigen Tools auf diesem Gebiet nicht kennen [69]. Auch Müller kommt in seiner Distant-Reading-Untersuchung von ca. 1.200 Monographien und Journal-Artikeln, die sich in der letzten Dekade mit Shakespeare beschäftigt haben, zu dem Schluss, dass es zwar eine kleine Gruppe von ForscherInnen gibt, welche umfassend digitale Werkzeuge einsetzten, deren Arbeit aber praktisch keinen Einfluss auf wichtige interdisziplinäre Trends hat [53]. Die fehlende Akzeptanz von digitalen Werkzeugen wird auch in der Interviewstudie von Rieger deutlich [56]. Nur vier der 45 befragten GeisteswissenschaftlerInnen gaben an, digitale Werkzeuge in ihrer Forschung einzusetzen. So postuliert die Autorin im Fazit ihres Artikels: „Many tools and techniques that are being associated with sophisticated digital practices, such as data mining or visualization, remain accessible and relevant to only a handful of scholars." In seinem Artikel „The Digital Humanities and Its Users“ merkt Edwards an, dass häufig nur die EntwicklerInnen eines Tools auch die späteren alleinigen NutzerInnen selbigens sind, obwohl es das Ziel von Entwicklungsteams ist eine breitere Zielgruppe anzusprechen [25].

Erste Ansätze zur Beleuchtung des Hintergrunds dieser fehlenden Nutzungsakzeptanz liefert Warwick [77]. In ihrem Paper beklagt sie die bisher mangelnde Einbeziehung der zukünftigen NutzerInnen bei der Entwicklung von Softwaretools. Ihrer Aussage nach wurde bis dato oft angenommen, dass technische Experten diejenigen seien, die wissen, wie digitale Werkzeuge aussehen sollten, was sie tun sollten und wie sie funktionieren sollten. Potenzielle NutzerInnen scheinen sich dieser Logik jedoch hartnäckig zu widersetzen [77]. Bradley verweist hingegen als Begründung darauf, dass „digitale“ und „traditionelle“ WissenschaftlerInnen dem Einsatz digitaler Werkzeuge in den Geisteswissenschaften unterschiedliche mentale Modelle zugrunde legen [9]. Einen weiteren Ansatz liefert Rieger, in dem sie auf die hohe tägliche Arbeitslast der Kollegen hinweist und argumentiert, dass diese nur bereit sind den entsprechenden Aufwand zur Durchdringung und Einbindung digitaler Werkzeuge auf sich nehmen, wenn damit auch für sie ein erkennbarer Mehrwert verbunden ist [56].

Wissenschaftliche Untersuchungen aus dem Bereich der Technologieakzeptanzforschung unter Anwendung einer oder mehrerer der in Abschnitt 2 aufgelisteten Modelle gibt es im Bereich der digitalen Geisteswissenschaften jedoch noch nicht. Einen ersten Hinweis hierfür liefern zahlreiche Metastudien über den Einsatz der wichtigsten Modelle TAM [40, 46, 47, 49, 50, 63, 70, 80, 81] und UTAUT $[24,44,75,78,79]$. Die in den Metastudien aufgelisteten Arbeiten untersuchen eine Vielzahl von unterschiedlichen Informationssystemen in unterschiedlichen Anwendungskontexten. Sowohl die Studien auf Basis des TAM als auch die Studien, welche die UTAUT zugrunde legen, können in die vier Anwendungskategorien (1) Kommunikationssysteme, (2) allgemeine Systeme, (3) Bürosysteme und (4) spezialisierte betriebliche Systeme eingeordnet werden (für UTAUT vgl.[78], für TAM vgl. [46]). Untersuchungen, die sich auf Anwendungen im Bereich Digital Humanities oder geisteswissenschaftliche Forschung im Allgemeinen beziehen, tauchen in den oben genannten Metastudien nicht auf.

Um diese Forschungslücke weiter zu untermauern, wurde eine Datenbankrecherche mit dem Suchstring: ((,TAM“ OR „UTAUT“ $O R$ “Technology Acceptance“ $O R$,TTF“ $O R$, „PB“ $O R$, „TRA“) AND 
(,dh“ $O R$ „,digital humanities“ $O R$,digitale Geisteswissenschaften “ $O R$ „Geisteswissenschaften“)) durchgeführt. Bei der Recherche wurden die folgenden Datenbanken berücksichtigt: (1) Web of Science ${ }^{5}$, (2) ACM Digital Library ${ }^{6}$, (3) ScienceDirekt ${ }^{7}$, (4) JSTOR ${ }^{8}$, (5) Google Scholar ${ }^{9}$ Hierbei konnte in keiner der genannten Datenbanken ein Text zur Technologieakzeptanz in den digitalen Geisteswissenschaften gefunden werden.

\subsection{Forschungsfrage}

In Abschnitt 1 wurde die große Menge der im Bereich DH entwickelten Anwendungen, sowie der Fördermittel, die hier von staatlicher Seite aus investiert werden, dargelegt. Es wurde ebenfalls argumentiert, dass die Nutzung dieser Softwaretools, was die Nutzungszahlen angeht, deutlich hinter den Erwartungen zurückbleiben. In Anbetracht dessen würde die Analyse der Akzeptanzfaktoren, welche die Nutzungsintentionen von digitalen Werkzeugen im DH Bereich beeinflussen, einen wichtigen Beitrag zur Beleuchtung dieses Problems leisten. Da es, wie oben erläutert, aktuell keine Forschung in diesem Bereich gibt, platziert sich die folgende Arbeit in eben dieser Forschungslücke mit der Fragestellung: Welche Faktoren beeinflussen die Akzeptanz der Nutzung von digitalen Tools in den Geisteswissenschaften?

\section{FORSCHUNGSDESIGN}

\subsection{Methodik}

Um die aufgeworfene Forschungsfrage zu beantworten, wird eine Akzeptanzfaktorenanalyse nach dem Vorbild von Davis [17] durchgeführt. Die empirische Umsetzung dieser Analyse wird operativ im Rahmen des BMBF geförderten Projekts „Humanist Computer Interaction " 10 durchgeführt. Im Rahmen dieses Projekts werden sechs Workshops mit insgesamt 50 TeilnehmerInnen an den Standorten Frankfurt, Münster, Stuttgart, Wien, Gent und Edinburgh durchgeführt. Die Workshops richten sich an Forschende aus den Geisteswissenschaften und bieten den TeilnehmerInnen eine erste Einführung in digitale Tools und digitale Arbeitsweisen. Diese Ausrichtung spricht ForscherInnen an, die potentiell Interesse an der Arbeit mit digitalen Tools auf ihrem Gebiet haben und nun vor der Entscheidung stehen solche Tools in zukünftigen Forschungsarbeiten einzubinden. Durch die hieraus resultierende Relevanz der Nutzungsentscheidung, für die Zielgruppe der Workshops, eignen sich diese TeilnehmerInnen auch sehr gut als Sample für die vorliegende Arbeit. Alle sechs Workshops sind identisch in vier Module aufgebaut. In drei dieser Modulen wird den TeilnehmerInnen jeweils ein digitales Tool in einem kurzen Vortrag vorgestellt. Anschließend haben die TeilnehmerInnen die Möglichkeit in sogenannten „Hands-On“-Sessions die Tools anhand vorgegebener Aufgabenstellungen selbst auszuprobieren und zu testen. Im Nachgang werden die Faktoren zur Bildung der Nutzungsintention sowie deren Determinanten in Form eines TAM-Fragebogens in Anlehnung an Venkatesh und Bala erhoben [72]. Dieses Setting orientiert

\footnotetext{
$\overline{{ }^{5} \text { http://wokinfo.com }}$

${ }^{6} \mathrm{https} / / / \mathrm{dl} . \mathrm{acm} . \mathrm{org}$

${ }^{7}$ https://sciencedirect.com

${ }^{8}$ https://jstor.org

${ }^{9}$ https://scholar.google.com

${ }^{10} \mathrm{https}$ //humanist.hs-mainz.de
}

Tabelle 1: Mapping Anwendungen und Forschungsaktivitäten

\begin{tabular}{lll}
\hline Anwendung & Kontext & Aktivität \\
\hline QAnnotate & Annotieren und & Annotieren, \\
& Kommentieren & Kontextualisieren \\
\hline nodegoad.net & Netzwerkanalyse & $\begin{array}{l}\text { Kontextualisieren, } \\
\text { Analysieren, } \\
\end{array}$ \\
& & Interpretieren \\
\hline Jupyter Notebook & Korpuslinguistische & Analysieren, \\
& $\begin{array}{l}\text { Analyse mit Python } \\
\text { Scripten }\end{array}$ & \\
& & \\
\hline
\end{tabular}

sich an der Original TAM-Studie. Dort argumentiert Davis, dass eine solch kurze Testphase auch dem Systemauswahlprozess, auf den das Modell zugeschnitten ist, in der Realität sehr nahe kommt [17]. Auch aktuellere Untersuchungen zeigen, dass gerade bei webbasierten Inhalten und Anwendungen die Nutzungsentscheidung erstaunlich schnell getroffen wird [48].

\subsection{Auswahl der Anwendungen}

Damit die oben beschriebene Untersuchung einen möglichst relevanten Ausschnitt des geisteswissenschaftlichen Arbeitsprozesses repräsentiert, wird die Auswahl, der in den Workshops verwendeten Anwendungen, auf Basis des von Stiller et al. entwickelten Arbeitsprozess für GeisteswissenschaftlerInnen getroffen [64]. Kern der Forschungsaktivitäten ist demnach ein Iterativer Zyklus aus (1) Annotieren, (2) Kontextualisieren, (3) Analysieren und (4) Interpretieren. Dieser Zyklus repräsentiert den Kern des Forschungsprozesses [36]. Es werden daher für die Untersuchungen Anwendungen ausgewählt, die in Summe alle vier Forschungsaktivitäten dieses Kerns abdecken.

Tabelle 1 listet die Anwendungen auf, welche für die Untersuchung verwendet werden und zeigt welche Forschungsaktivitäten durch diese abgedeckt werden.

„QAnnotate“ ist eine Anwendung zum Annotieren und Kommentieren von historischen Texten. Diese wurde im Rahmen des BMBFProjekts „Humanist-Computer Interaction “ von der TU-Darmstadt entwickelt. Die Software richtet sich vor allem an Einsteiger, da sie keine Vorkenntnisse auf dem Gebiet TEI/XML ${ }^{11}$ Codierung voraussetzt und die NutzerInnen beim Annotieren nicht mit dem erzeugten Code in Berührung kommen. Bei den Forschungsaktivitäten deckt QAnnotate die Schritte Annotieren und Kontextualisieren ab. In der Hands-On Session zu dieser Anwendung bekommen die WorkshopteilnehmerInnen die Aufgabe selbst einige Annotationen und Kommentare zu erstellen, sowie einen fehlenden Eintrag in der hinterlegten Datenbank zu ergänzen.

„nodegoad.net“ ist eine webbasierte Netzwerkanalyse- und Visualisierungsumgebung mit integriertem Datenmanagement [71]. Sie ermöglicht es der NutzerIn Daten, welche zuvor (z.B. mit QAnnotate) angereichert und annotiert wurden, in Form von Netzwerken $\mathrm{zu}$ visualisieren und $\mathrm{zu}$ analysieren. Auch diese Anwendung ist

\footnotetext{
${ }^{11}$ https://tei-c.org
} 
einsteigerfreundlich, da die Netzwerke über ein klickbares Interface und nicht über die Eingabe von Code erzeugt werden. Die WorkshopteilnehmerInnen bekommen in der Hands-On Session die Aufgabe ein Netzwerk aus zuvor annotierten Daten zu erstellen und mit dessen Hilfe das Verwandschaftsverhältnis zwischen zwei Personen zu identifizieren.

Bei der webbasierten Anwendung „fupyter Notebook“ handelt es sich um eine interaktive Entwicklungsumgebung für die Programmiersprache Python, welche Live-Code, Visualisierungen und narrativen Text miteinander verbindet [54]. Die Anwendung ist weit verbreitet im Bereich Data-Science, und wird auch in den Digital Humanities eingesetzt. Bei dieser Anwendung können die TeilnehmerInnen während der Hands-On Session Schritt für Schritt den vorgegebenen Code ausführen und durch die Manipulation von Attributen im Code die Ergebnisse nach ihren Wünschen manipulieren.

\section{ERMITTLUNG DER EINFLUSSFAKTOREN}

\subsection{Erhebung}

Bei der empirischen Durchführung der Untersuchung konnten, entsprechend der obigen Ausführungen, drei Anwendungen von jeweils ca. 50 Probanden getestet werden. In Summe wurde so ein bereinigter Datensatz mit 149 Fällen erhoben. Bei den TeilnehmerInnen der Untersuchung handelt es sich vornehmlich um GeisteswissenschaftlerInnen aus den Geschichts- und Literaturwissenschaften.

In der nun folgenden statistische Analyse wird zunächst evaluiert, wie groß der Einfluss der beide Hauptkonstrukte Perceived Usefulness (PU) und Perceived Ease of Use (PEOU) auf die Nutzerakzeptanz ist. Weiter wird anschließend ermittelt, wie groß der Einfluss der einzelnen Determinanten Subjective Norm (SN), Image (IMG), fob Relevance (REL), Output Quality (OUT) und Result Demonstrability (RES) auf Perceived Usefulness und Computer Self-Efficacy (CSE), Perceptions of External Control (PEC), Computer Anxiety (CANX), Computer Playfulness (CPLAY), Perceived Enjoyment (ENF) und auf Perceived Ease of Use ist.

Ist die Einflussgröße der einzelnen Konstrukte des Modells bekannt, kann daraus abgeleitet werden, welche Determinanten das größte Potential zur Einflussnahme auf die Nutzungsakzeptanz bieten. In anschließenden Untersuchungen können auf dieser Grundlage Handlungsstrategien und Implikationen für die Praxis aus den Bereichen User Centered Design (UCD) und Human Computer Interaction (HCI) abgeleitet werden. Mit Hilfe dieser Handlungsstrategien können SoftwareentwicklerInnen ihre Anwendungen optimieren um die Akzeptanz bei den NutzerInnen zu steigern.

\subsection{Auswahl des statistischen Verfahrens}

Zur Analyse der, im Rahmen der Workshops erhobenen Daten wird das statistische Verfahren der Strukturgleichungsmodellierung, Structural Equation Modeling (SEM), angewendet. Mit Hilfe dieses Verfahrens ist es möglich, nomologische Netzwerke zu modellieren, indem theoretische Konzepte durch Konstrukte ausgedrückt und diese Konstrukte über ein Strukturmodell verbunden werden um so ihre Beziehungen zu untersuchen [6]. Hierbei kann zwischen dem kovarianzbasierten Verfahren, Covariance-Based Structural
Equation Modeling (CB-SEM) und dem varianzbasierten Verfahren unterschieden werden. Ein in der Wirtschaftsinformatik weit verbreitetes varianzbasiertes Verfahren ist das Partial Least Square Structural Equation Modeling (PLS-SEM) [61]. Während kovarianzbasierte Verfahren die Diskrepanz zwischen der empirischen und der modellimplizierten Varianz-Kovarianz-Matrix der beobachtbaren Indikatoren minimieren, um die Modellparameterschätzungen zu erhalten, erstellen varianzbasierte Schätzer Linearkombinationen der Indikatoren stellvertretend für die theoretischen Konzepte und schätzen anschließend die Modellparameter [5]. In der Vergangenheit wurde immer wieder diskutiert wann die Anwendung von PLS-SEM oder CB-SEM angemessener ist [31, 38, 43, 51, 52, 58]. Diese Diskussion hat sich mittlerweile etwas verlagert, da sich PLS-SEM als eigenständige Methode etabliert hat [57, 60]. In der vorliegenden Arbeit wird PLS-SEM verwendet. Diese Entscheidung begründet sich aus der Empfehlung von Hair, Risher, Sarstedt und Ringle [34], PLS-SEM anzuwenden wenn, wie in der vorliegenden Arbeit der Fall, ...

(1) ... es bei der Analyse darum geht einen theoretischen Rahmen aus einer Vorhersageperspektive zu testen.

(2) ... das Strukturmodell komplex ist und viele Konstrukte, Indikatoren und/oder Modellbeziehungen enthält.

(3) ... das Forschungsziel darin besteht die zunehmende Komplexität besser zu verstehen, indem theoretisch etablierte Theorien erweitert oder auf neue Gebiete übertragen werden.

(4) ... die Stichprobengröße aus methodischen Gründen eingeschränkt ist.

(5) ... die Stichprobenverteilung ungünstig ist, z.B. bei fehlender Normalverteilung.

Zur Schätzung des Modells wurde die Software ADANCO ${ }^{12}$ verwendet. Da es sich bei allen Faktoren um reflektive Konstrukte handelt, werden diese mit dem consistent PLS-Verfahren (PLSc) geschätzt. Dieses Verfahren ermöglicht die konsistente Schätzung von Modellen mit latenten Variablen, hierbei werden die geringer ausfallenden Korrelationen der Konstrukt-Ladungen korrigiert $[21,22]$.

\subsection{Stichprobengröße}

Mittlerweile ist man sich einig darüber, dass Faustformeln zur Bestimmung der Mindeststichprobengröße, wie z.B. die viel zitierte 10-Fach-Regel, welche besagt, dass die minimale Stichprobengröße das Zehnfache der maximalen Anzahl von Pfeilspitzen betragen sollte, die auf eine der latente Variable im PLS-Pfadmodell zeigen [3], nicht mehr angemessen sind [33, 45, 59]. Hair [33] empfiehlt daher die minimale Stichprobengröße auf Basis einer statistischen Teststärkeanalyse durchzuführen und verweist dabei auf die Teststärke Tabellen von Cohen [15]. Nach Cohen benötigt man um eine Teststärke von $80 \%$ zu erreichen, ausgehend von einem Signifikanzniveau von $5 \%$, einem minimalen $R^{2}$ von 0,1 und, wie im verwendeten TAM maximal sechs unabhängigen Variablen pro Konstrukt, 130 Beobachtungen. Demnach ist die vorliegende Strichprobe mit 149 Fällen ausreichend groß um statistisch signifikante Effekte zu ermitteln.

\footnotetext{
${ }^{12}$ www.composite-modeling.com
} 


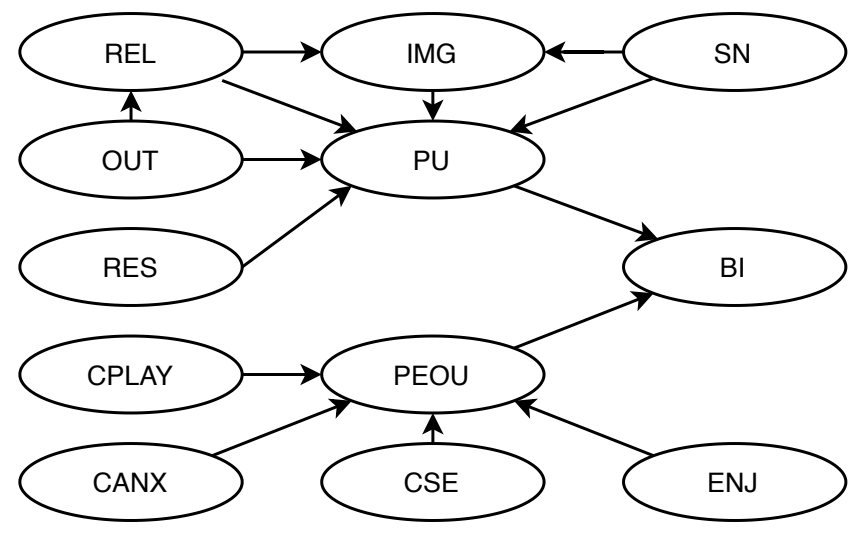

Abbildung 1: Größtmögliches Modell in Anlehnung an das TAM

Tabelle 2: Ergebnisse der konfirmatorischen Faktorenanalyse zur Evaluation der Anpassungsgüte des Messmodells

\begin{tabular}{llll}
\hline & Wert & HI95 & Beurteilung \\
\hline SRMR & 0,0908 & 0,0934 & akzeptabel \\
$\mathrm{d}_{U L S}$ & 7,8032 & 8,2438 & akzeptiert \\
$\mathrm{d}_{G}$ & 5,4866 & 7,0341 & akzeptiert \\
\hline
\end{tabular}

\subsection{Modellentwicklung}

Im ersten Schritt wurde versucht möglichst alle abgefragten Konstrukte in Anlehnung an das TAM in ein Modell zu integrieren. Analog zum Vorgehen von Venkatesh und Bala [72] wurden alle Konstrukte unter der Verwendung von reflektiven Indikatoren modelliert. Um ein gültiges Modell zu erhalten, mussten zunächst das Konstrukt Perceived External Control und der Indikator Output Quality 2 verworfen werden, da diese in der vorliegenden Konstellation jeweils zu einem sogenannten Heywoodcase führen würden [37]. Das hieraus resultierende Modell ist in Abbildung 1 dargestellt.

Bereits hier ist erkennbar, dass sich der TAM-Kern (Perceived Ease of Use und Perceived Usefulness beeinflussen die Behavioral Intention) auch auf die Zielgruppe der Geisteswissenschaftler anwenden lässt. Weiterhin zeigt die Grafik jedoch auch deutlich, dass das Modell mit der Vielzahl der aktuell vorhandenen Faktoren eine kaum signifikante Aussagekraft hat. Dies bestätigt auch die Anpassungsgüte (engl. goodness of fit) des Modells. Zur Beurteilung dieser zeigen Tabelle 2 und Tabelle 3 die Ergebnisse der Modellpassungsevaluation für das Mess- und das Schätzmodell mittels konfirmatorischer Faktorenanalyse.

Tabelle 2 zeigt eine gute Passung des Modells. Der Wert für die standardisierte Residualdiskrepanzwurzel (engl. Standardized Root Mean Residual, SRMR) liegt zwar knapp über dem empfohlenen Schwellenwert von 0,08 [38, 41], aber immer noch unter dem 95\%Quantil der entsprechenden Referenzverteilung (HI95), was nach Benitez et al. [5] der bessere Schwellenwert für die Anpassungsgüte darstellt. Der quadrierte euklidische Abstand (engl. squared Euclidean distance, $\mathrm{d}_{U L S}$ ) sowie der geodätische Abstand (engl. geodesic distance, $d_{G}$ ) liegen beide ebenfalls unter dem 95\%-Quantil ihrer
Tabelle 3: Ergebnisse der konfirmatorischen Faktorenanalyse zur Evaluation der Anpassungsgüte des Schätzmodells

\begin{tabular}{lllll}
\hline & Wert & HI95 & HI99 & Beurteilung \\
\hline SRMR & 0,1180 & 0,1006 & 0,1090 & nicht akzeptiert \\
$\mathrm{d}_{U L S}$ & 13,1688 & 9,5790 & 11,2451 & nicht akzeptiert \\
$\mathrm{d}_{G}$ & 5,8333 & 7,0959 & 8,4475 & akzeptiert \\
\hline
\end{tabular}

Tabelle 4: Ergebnisse der konfirmatorischen Faktorenanalyse zur Evaluation der Anpassungsgüte des optimierten Messmodells

\begin{tabular}{llll}
\hline & Wert & HI95 & Beurteilung \\
\hline SRMR & 0,0417 & 0,0507 & akzeptiert \\
$\mathrm{d}_{U L S}$ & 0,4016 & 0,5939 & akzeptiert \\
$\mathrm{d}_{G}$ & 0,5410 & 0,7789 & akzeptiert \\
\hline
\end{tabular}

Referenzverteilung (HI95), was auch bei diesen beiden Messwerten den Schwellenwert bildet [5]. Das verwendete Messmodell kann demnach für die vorliegende Untersuchung angenommen werden.

Betrachtet man jedoch Tabelle 3 wird deutlich, dass dies für das Schätzmodell nicht der Fall ist. Der Wert für $\mathrm{d}_{G}$ wäre zwar akzeptabel, der SRMR-Wert liegt jedoch deutlich über dem Schwellenwert von 0,08 und auch das 95\%-Quantil der Referenzverteilung wird vom SRMR- und $\mathrm{d}_{U L S}$-Werte überschritten. Auch eine Betrachtung des 99\%-Quantil der Referenzverteilung (HI99) zeigt, dass die Diskrepanzen hier ebenfalls zu groß sind. Es kann demnach davon ausgegangen werden, dass das Schätzmodell nicht die Realität abbildet. Aus diesem Grund wurde das Modell weiter optimiert um ein Modell mit einer akzeptablen Anpassungsgüte zu erhalten. Um dies zu erreichen, wurden die Konstrukte ohne oder mit sehr geringem Einfluss schrittweise aus dem Modell entfernt. Weiterhin wurden die Konstrukte um Indikatoren mit geringer Ladung bereinigt. Das finale Modell ist in Abbildung 2 abgebildet.

In Tabelle 4 ist zu sehen, dass auch das Messmodell für diese optimierte Variante eine hohe Anpassungsgüte aufweist. Die Werte für SRMR, $\mathrm{d}_{U L S}$ und $\mathrm{d}_{G}$ liegen alle drei unterhalb der bereits weiter oben erörterten Schwellenwerte. Wie bereits beim Ausgangsmodell liefern diese Daten hiermit den empirischen Beweis, dass die gemessenen Konstrukte in dieser Form auch existieren und aus den gewählten Indikatoren abgeleitet werden können.

Auch für das Schätzmodell liefert die konfirmatorische Faktorenanalyse sehr gute Modell-Werte (Tabelle 5). Hier liegen ebenfalls alle drei Indikatoren (SRMR, $\mathrm{d}_{U L S}, \mathrm{~d}_{G}$ ) unterhalb des Schwellenwerts. Die Evaluation der Anpassungsgüte liefert somit einen empirischen Beweise für das postulierte Modell. Es ist demnach möglich, dass die empirischen Daten aus einer Welt stammen die, wie von diesem Modell theoretisiert, funktioniert [5].

\subsection{Validitäts- und Reliabilitätsprüfung}

Um die Reliabilität des Modells sicherzustellen wird im Folgenden die Evaluation von (1) Konstruktreliabilität, (2) Konvergenzvalidität, (3) Indikatorreliabilität und (4) Diskriminanzvalidität nach den Richtlinien von Benitez et al. [5] durchgeführt. 
Tabelle 5: Ergebnisse der konfirmatorischen Faktorenanalyse zur Evaluation der Anpassungsgüte des optimierten Messmodells

\begin{tabular}{llll}
\hline & Wert & HI95 & Beurteilung \\
\hline SRMR & 0,0618 & 0,0658 & akzeptiert \\
$\mathrm{d}_{U L S}$ & 0,8822 & 1,0002 & akzeptiert \\
$\mathrm{d}_{G}$ & 0,5640 & 0,8340 & akzeptiert \\
\hline
\end{tabular}

Tabelle 6: Evaluation von Konstruktreliabilität und Konvergenzvalidität

\begin{tabular}{llll}
\hline Konstrukte & $\rho_{A}$ & $\rho_{c}$ & AVE \\
\hline BI & 0,927 & 0,925 & 0,804 \\
PEOU & 0,880 & 0,878 & 0,706 \\
PU & 0,944 & 0,943 & 0,806 \\
OUT & 0,794 & 0,775 & 0,635 \\
ENJ & 0,948 & 0,945 & 0,851 \\
CANX & 0,805 & 0,802 & 0,576 \\
SN & 0,841 & 0,802 & 0,586 \\
\hline
\end{tabular}

Zur Bewertung der Konstruktvalidität wird häufig die kongenerische Reliabilität $\rho_{c}$ von Jöreskog [42] herangezogen. Werte ab 0,7 gelten hier als akzeptabel [34], sie sollten jedoch den Wert 0,95 nicht überschreiten, da dies darauf hindeuten würde, dass die Indikatoren redundant sind, was die Konstruktreliabilität reduzieren würde $[20,23]$. Wie in Tabelle 6 zu erkennen, liegen fast alle oberhalb von 0,8 und überschreiten die obergrenze von 0,95 nicht. Eine etwas weniger liberale Alternative stellt der Indikator $\rho_{A}$ von Dijkstra und Henseler [22] dar. Ab einem Schwellenwert von 0,7 gilt die Konstruktreliabilität als angemessen, da hier $50 \%$ der Varianz in den Konstruktwerten erklärt wird. Im vorliegenden Sample liegt der niedrigste Wert bei 0,794, wodurch sich die Konstruktreliabilität bestätigt.

Zur Bewertung der Konvergenzvalidität wird typischerweise die durchschnittlich erfasste Varianz (engl. average variance extracted, AVE) herangezogen. Ein Wert über 0,5 gilt als empirische Evidenz für Konvergenzvalidität, da die entsprechende latente Variable dann mehr als die Hälfte der Varianz der zugehörigen Indikatoren erklärt [27]. Die Werte in Tabelle 6 liegen alle über 0,5 und bestätigen damit die Konvergenzvalidität des vorliegenden Modells.

Die Indikatorreliabilität kann über die Höhe der Faktorladungen bestimmt werden. Im Idealfall sollten die Faktorladungen über 0,707 liegen. Da dieser Wert impliziert, dass mehr als 50\% der Varianz innerhalb eines Indikators durch die entsprechende latente Variable erklärt wird. Bis auf zwei Indikatoren liegen diese alle über dem Schwellenwert von 0,707 (siehe Tabelle 7). Output Quality 3 liegt mit einer Faktorladungen von 0,699 jedoch nur geringfügig unter diesem Wert. Etwas problematischer ist der Wert von Subjective Norm. Er liegt bei 0,528. Eine solch, relativ geringe Abweichung bei den Faktorladungen kann jedoch bei den vorliegenden guten Konstruktreliabilitäs- und Konvergenzvaliditätswerten des Modells vernachlässigt werden [5].
Tabelle 7: Faktorladungen der Indikatoren

\begin{tabular}{|c|c|c|c|c|c|c|c|}
\hline Indik. & BI & CANX & ENJ & OUT & PEOU & $\mathrm{PU}$ & SN \\
\hline BI1 & 0,893 & & & & & & \\
\hline BI2 & 0,946 & & & & & & \\
\hline BI3 & 0,848 & & & & & & \\
\hline CANX1 & & 0,707 & & & & & \\
\hline CANX2 & & 0,798 & & & & & \\
\hline CANX3 & & 0,769 & & & & & \\
\hline ENJ1 & & & 0,973 & & & & \\
\hline ENJ2 & & & 0,933 & & & & \\
\hline ENJ3 & & & 0,859 & & & & \\
\hline OUT1 & & & & 0,885 & & & \\
\hline OUT3 & & & & 0,699 & & & \\
\hline PEOU1 & & & & & 0,882 & & \\
\hline PEOU3 & & & & & 0,814 & & \\
\hline PEOU4 & & & & & 0,824 & & \\
\hline PU1 & & & & & & 0,950 & \\
\hline PU2 & & & & & & 0,872 & \\
\hline PU3 & & & & & & 0,889 & \\
\hline PU4 & & & & & & 0,878 & \\
\hline SN1 & & & & & & & 0,838 \\
\hline SN2 & & & & & & & 0,881 \\
\hline SN4 & & & & & & & 0,528 \\
\hline
\end{tabular}

Tabelle 8: Diskriminanzvalidität Fornell-Larcker

\begin{tabular}{lccccccc}
\hline Konstr. & BI & PEOU & PU & OUT & ENJ & CANX & SN \\
\hline BI & 0,804 & & & & & & \\
PEOU & 0,231 & 0,706 & & & & & \\
PU & 0,489 & 0,089 & 0,806 & & & & \\
OUT & 0,243 & 0,134 & 0,342 & 0,635 & & & \\
ENJ & 0,293 & 0,247 & 0,171 & 0,266 & 0,851 & & \\
CANX & 0,168 & 0,162 & 0,067 & 0,069 & 0,114 & 0,576 & \\
SN & 0,267 & 0,141 & 0,360 & 0,146 & 0,056 & 0,032 & 0,586 \\
\hline
\end{tabular}

Quadrierte Konstruktkorrelationen; AVE auf der Diagonalen

Bei gegebener Diskriminanzvalidität sind zwei latente Variablen, die zwei unterschiedliche theoretische Konzepte repräsentieren, statistisch ausreichend unterschiedlich. Überprüft werden kann dies entweder mit Hilfe des Fornell-Larcker Kriteriums [27] oder des Heterotrait-Monotrait Verhältnis der Korrelationen (HTMT) [39]. Das Fornell-Larcker Kriterium besagt, dass die quadrierten Konstruktkorellationen nicht größer sein dürfen als deren AVE. Tabelle 8 bestätigt, dass dies beim vorliegenden Modell der Fall ist. Der HTMT-Wert soll nach Henseler et al. [39] möglichst unter 0,85 liegen. Tabelle 9 zeigt, dass das Modell auch dieses Kriterium erfüllt. Die Diskriminanzvalidität ist somit auch empirisch bestätigt.

Somit können für das vorliegende Modell, Konstruktreliabilität, Konvergenzvalidität, Indikatorreliabilität und Diskriminanzvalidität bestätigt werden. 
Tabelle 9: Diskriminanzvalidität HTMT

\begin{tabular}{lrrrrrr}
\hline Konstr. & BI & PEOU & PU & OUT & ENJ & CANX \\
\hline PEOU & 0,480 & & & & & \\
PU & 0,701 & 0,298 & & & & \\
OUT & 0,497 & 0,374 & 0,589 & & & \\
ENJ & 0,541 & 0,499 & 0,414 & 0,525 & & \\
CANX & 0,415 & 0,404 & 0,261 & 0,267 & 0,341 & \\
SN & 0,534 & 0,398 & 0,616 & 0,404 & 0,240 & 0,183 \\
\hline
\end{tabular}

Tabelle 10: Strukturmodellwerte

\begin{tabular}{lccrc}
\hline Effekte & Koeff. & T & p & $f^{2}$ \\
\hline PU $\rightarrow$ BI & 0,610 & 8,466 & 0,000 & 0,788 \\
PEOU $\rightarrow$ BI & 0,299 & 4,467 & 0,000 & 0,189 \\
SN $\rightarrow$ PU & 0,440 & 6,264 & 0,000 & 0,337 \\
SN $\rightarrow$ PEOU & 0,249 & 3,082 & 0,001 & 0,091 \\
OUT $\rightarrow$ PU & 0,417 & 4,658 & 0,000 & 0,301 \\
CANX $\rightarrow$ PEOU & $-0,237$ & $-2,859$ & 0,002 & 0,078 \\
ENJ $\rightarrow$ PEOU & 0,359 & 4,214 & 0,000 & 0,174 \\
\hline
\end{tabular}

\subsection{Bewertung des Strukturmodells}

Zur Bewertung des Strukturmodells werden im folgenden die Pfadkoeffizienten und deren Signifikanzniveau, die Effektgrößen und die Determinationskoeffizienten des Modells betrachtet. Eine grafische Repräsentation des Modells zeigt Abbildung 2. Wie in dieser Abbildung zu sehen, sind die Pfadkoeffizienten alle mindestens auf einem Signifikanzniveau von 5\% signifikant. Die Werte der Pfadkoeffizienten liegen dabei in einem akzeptablen Bereich zwischen 0,237 und 0,610 (vgl. Tabelle 10).

Als Messwert für die Effektstärke wird Cohens $f^{2}$ verwendet [14]. Diese Messwerte finden sich ebenfalls in Tabelle 10. Die Effektstärke kann in schwach $(0,02$ bis 0,150$)$ mittel $(0,150$ bis 0,350$)$ und groß $(\geq 350)$ eingestuft werden. Die meisten Effektstärken des Modells können dem mittleren Bereich zugeordnet werden. Lediglich die Effektstärken von Subjective Norm und Computer Anxiety auf Perceived Ease of Use liegen im schwachen Bereich. Im Gegenzug kann die Effektstärke von Perceived Usefulness auf die Behavioral Intention als groß eingestuft werden.

Die Determinationskoeffizienten des Modells sind in Tabelle 11 abgebildet. Der Determinationskoeffizient $R^{2}$ gibt den Anteil der Varianz an, der in einem latenten Konstrukt erklärt wird und gibt somit Aufschluss über die Vorhersagekraft eines Modells [4]. Das $R^{2}$ stellt mit 0,570 auch im Vergleich zu anderen Untersuchungen wie z.B. der original Modellkonstruktionsstudie von Venkatesh und Bala [72] einen sehr guten Wert dar. Gleiches gilt für das $R^{2}$ von Perceived Usefulness, welches mit 0,508 ebenfalls mehr als die Hälfte der Varianz erklärt. Im Vergleich fällt das $R^{2}$ von Perceived Ease of Use mit 0,367 etwas geringer aus. In Anbetracht dessen, dass es sich hierbei aber um eine der ersten Untersuchungen dieser Art auf dem Gebiet der Geisteswissenschaften handelt kann dieser durchaus als akzeptabel angesehen werden.
Tabelle 11: Determinationskoeffizienten

\begin{tabular}{llll}
\hline Konstrukt & BI & PEOU & PU \\
\hline$R^{2}$ & 0,570 & 0,367 & 0,508 \\
\hline
\end{tabular}

\subsection{Ergebnisinterpretation}

Wie im ursprünglichen TAM von Davis [17] und allen später folgenden Erweiterungen, bilden auch in dem vorliegenden Modell mit Fokus auf die Akzeptanz von digitalen Werkzeugen in den Geisteswissenschaften, PEOU und PU die beiden Haupteinflussfaktoren für die Behavioral Intention. Es kann also festgehalten werden, dass das TAM im Kern auch auf den Bereich der Geisteswissenschaften übertragbar ist. Was die Effektstärken und Pfadkoeffizienten von diesen beiden Faktoren angeht, so ist der Einfluss von PU mit einem $f^{2}$ von 0,788 und einem Pfadkoeffizienten von 0,610 sehr hoch und deutlich höher als der Einfluss von PEOU mit einem $f^{2}$ von 0,189 einem Pfadkoeffizienten von 0,299.

Mit Blick auf die HCI bedeutet dies, dass der funktionale Aspekt der Nützlichkeit (PU) eine weit größere Rolle spielt als die Gebrauchstauchlichkeit (PEOU). Ein digitales Werkzeug welches seinen Zweck gut erfüllt und dafür Schwächen bei der Bedienbarkeit aufweist, wird vermutlich eher verwendet als ein Werkzeug mit schöner Bedienoberfläche aber fehlendem Funktionsumfang. Diese Ergebnisse sind konsistent mit den ursprünglichen TAM-Studien $[17,72,73]$. Auch dort ist der Effekt von PU stärker ausgeprägt als der von PEOU. Zudem wurde hier auch ein Effekt zwischen den beiden Faktoren gemessen. Es wäre demnach zu erwarten, dass die Gebrauchstauchlichkeit auch einen Einfluss auf die Nützlichkeit der digitalen Werkzeuge hat. Ein solcher Effekt konnte jedoch bei der vorliegenden Untersuchung im geisteswissenschaftlichen Kontext nicht gemessen werden. Für die Geisteswissenschaftler in unserem Sample hat die Einfachheit der Bedienung somit keinen messbaren Einfluss auf die Nützlichkeit des Werkzeuges. Die Bedeutung des Faktors PEOU tritt somit weiter in den Hintergrund. Weiterhin liefert das Modell auch Informationen darüber welche Faktoren wiederum die beiden Hauptkonstrukte PU und PEOU maßgeblich beeinflussen. Entsprechend dem vorliegenden Modell kann festgestellt werden, dass für die befragte Zielgruppe die Nützlichkeit PU von digitalen Werkzeugen von der Qualität der Ergebnisse, die mit diesen Werkzeugen produziert werden können (OUT) und vom sozialen Umfeld der ForscherInnen (SN) bestimmt wird. Auf Grund des hohen $R^{2}$ von PU in Höhe von 0,508 und einer ausgewogenen Effektstärke der bestimmenden Konstrukte im oberen Mittelbereich $\left(f^{2} 0,337\right.$ und 0,301$)$, kann davon ausgegangen werden, dass es sich hier um die beiden primären Faktoren handelt, welche das Konstrukt PU beeinflussen.

Weiterhin kann festgestellt werden, dass die Einfachheit der Benutzung PEOU von digitalen Werkzeugen für die an der Untersuchung teilnehmenden WissenschaftlerInnen durch positive Nutzungserfahrungen (ENJ), dem sozialen Umfeld (SN) und der Angst beim Umgang mit diesen Werkzeugen (CANX) bestimmt wird. Hierbei ist anzumerken, dass ENJ und CANX auch bereits im ursprünglichen TAM in dieser Form existieren, der Einfluss von SN auf PEOU liegt hingengen nur in dem hier entwickelten Modell 


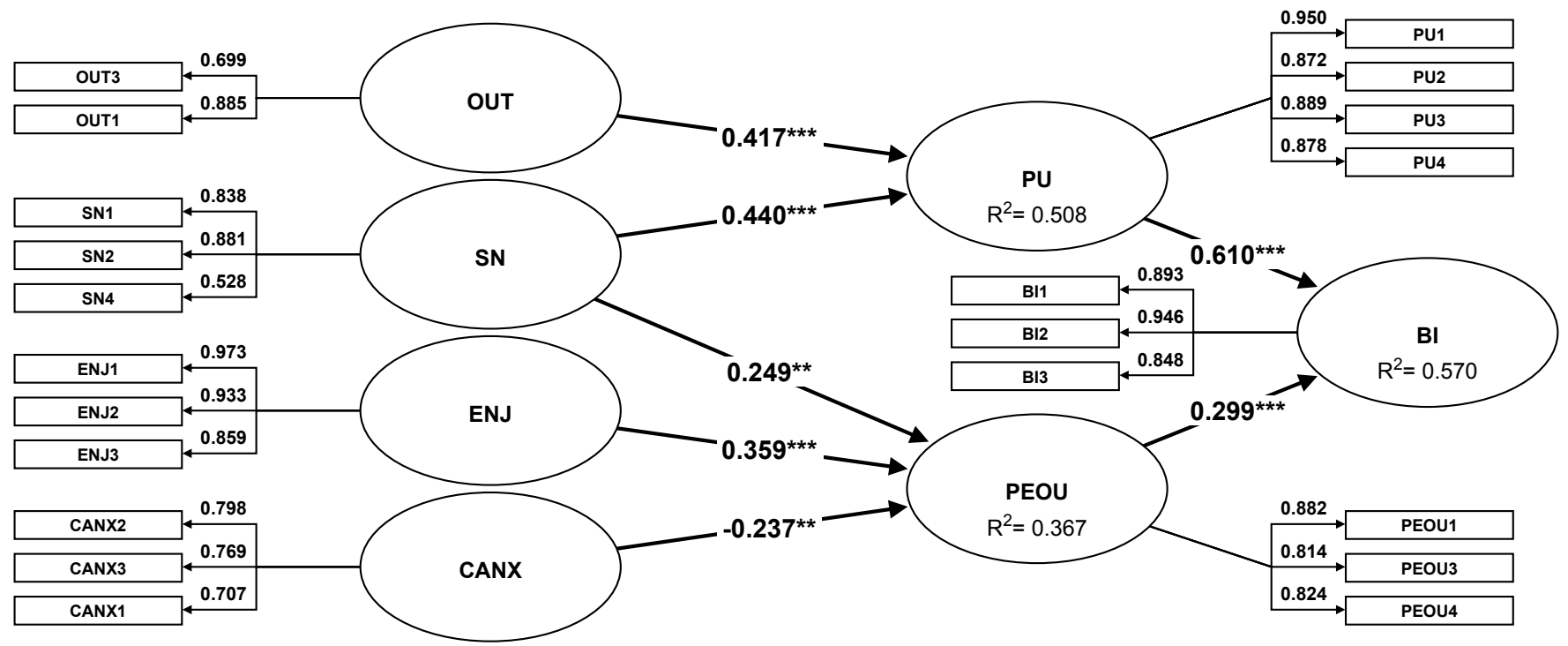

Abbildung 2: Optimiertes Modell

vor und ist somit als zielgruppenspezifisch anzusehen. Was die Effektstärken angeht, handelt es sich hierbei mit einem $f^{2}$ von 0,091 auch um einen sehr schwachen Effekt. Auch die Angst vor dem Umgang mit den digitalen Werkzeugen hat lediglich ein $f^{2}$ von 0,078 und somit ebenfalls eine sehr geringe Effektstärke. Daraus lässt sich ableiten, dass positive Erfahrungen bei der Nutzung der Werkzeuge mit einem $f^{2}$ von 0,174 den größten Einfluss auf die PEOU haben. Das relativ geringe $R^{2}$ von 0,367 lässt jedoch vermuten, dass es für diese Zielgruppe noch weitere Faktoren gibt, welche PEOU bestimmen.

$\mathrm{Da}$, wie weiter oben dargelegt, die funktionale Nützlichkeit gegenüber der Gebrauchstauglichkeit die größere Bedeutung hat, nehmen auch die beiden hier anknüpfenden Faktoren, Ergebnisqualität und soziales Umfeld, eine wichigere Rolle ein als die CANX und ENJ. Da im vorliegenden Modell auch einen direkten Einfluss SN auf die Gebrauchstauchlichkeit existiert, hat dieser Faktor insgesamt den größten Einfluss. Für die befragten GeisteswissenschaftlerInnen ist es somit in erster Linie wichtig ob die Verwendung eines digitalen Werkzeugs von Menschen, die im beruflichen Kontext Einfluss auf sie haben oder die für sie wichtig sind, die Verwendung eines digitalen Werkzeugs befürworten. Weiterhin bezieht das Konstrukt SN auch mit ein, ob die übergeordnete Institution oder Organisationseinheit die Verwendung des digitalen Werkzeugs unterstützt.

Ob GeisteswissenschaftlerInnen die Verwendung eines digitalen Werkzeugs als angenehm empfinden oder die Verwendung sogar Spaß macht (ENJ), hat zwar auch einen signifikanten Einfluss auf dessen Adaption, dieser Einfluss ist im Vergleich zum Sozialen Umfeld (SN) und der Qualität der Ergebnisse (OUT) jedoch nachrangig. Auch die den GeisteswissenschaftlerInnen oft vorgeworfene fehlende Technikaffinität oder vorliegende Bedenken mit dem Umgang von Computern (CANX) hat zwar einen messbaren Einfluss auf die Akzeptanz von digitalen Werkzeugen, im Vergleich zu den beiden oben genannten Hauptfaktoren, fällt dieser Effekt jedoch eher gering aus.

\subsection{Fazit}

Die obigen Ausführungen stellen einen ersten Schritt zur Beantwortung der eingangs gestellten Frage nach der fehlenden Nutzerakzeptanz von digitalen Werkzeugen da. Die Ergebnisse legen nahe, dass sich die fehlende Akzeptanz eher aus dem sozialen und institutionellen Umfeld der GeisteswissenschaftlerInnen sowie der Qualität der gelieferten Ergebnisse als aus einer möglicherweise fehlenden nutzerfreundlichen Bedienbarkeit resultiert. Um die Akzeptanz von digitalen Werkzeugen zu erhöhen sollte daher primär die Verbesserung dieser beiden Faktoren und die damit zusammenhängende funktionale Nützlichkeit angestrebt werden. Weiterhin hat die Usability der digitalen Werkzeuge einen signifikanten Einfluss auf die Nutzungsakzeptanz und bietet daher ebenfalls Ansatzmöglichkeiten zur Verbesserung. Diese sollten mit sekundärer Priorität verfolgt werden, da sie, entsprechen der obigen Ergebnisse, weniger effizient sind. Hier sollten vorallem die Angst vor dem Umgang mit Computern bzw. die Bedenken der Nutzer hierbei, sowie eine möglichst angenehme Bedienung im Vordergrund stehen.

In einem nächsten Schritt kann nun auf den Ergebnissen dieser Arbeit aufgebaut werden. Da hier die Einflussfaktoren für die Nutzungsakzeptanz von digitalen Werkzeugen für GeisteswissenschaftlerInnen ermittelt wurden, können in einem nächste Schritt, im Rahmen der Design-Science Forschung, gezielt Guidelines und Handlungsempfehlungen zur Steigerung der Nutzungsakzeptanz entwickelt und überprüft werden.

\section{LITERATUR}

[1] Icek Ajzen. 1991. The Theory of Planned Behavior. Organizational Behavior and Human Decision Processes 50, 2 (Dec. 1991), 179-211. https://doi.org/10.1016/07495978(91)90020-T 
[2] Arno Anzenbacher. 2010. Einführung in die Philosophie (neuausgabe, 7. auflage, (14. gesamtauflage) ed.). Herder, Freiburg im Breisgau Basel Wien.

[3] Donald Barclay, Christopher Higgins, and Ronald Thompson. 1995. The Partial Least Squares (PLS) Approach to Casual Modeling: Personal Computer Adoption Ans Use as an Illustration. Technology Studies 1995, 2 (1995), 286-309.

[4] Jan-Michael Becker, Arun Rai, and Edward Rigdon. 2013. Predictive Validity and Formative Measurement in Structural Equation Modeling: Embracing Practical Relevance. In Proceedings of the International Conference on Information Systems (ICIS). Milan, 20.

[5] Jose Benitez, Jörg Henseler, Ana Castillo, and Florian Schuberth. 2020. How to Perform and Report an Impactful Analysis Using Partial Least Squares: Guidelines for Confirmatory and Explanatory IS Research. Information \& Management 57, 2 (March 2020), 103168. https://doi.org/10.1016/j.im.2019.05.003

[6] Kenneth A. Bollen. 1989. A New Incremental Fit Index for General Structural Equation Models. Sociological Methods \& Research 17, 3 (Feb. 1989), 303-316. https://doi.org/10.1177/0049124189017003004

[7] Christine L. Borgman. 2010. The Digital Future Is Now: A Call to Action for the Humanities. Digital Humanities Quarterly 003, 4 (March 2010).

[8] Edmund Addison Bowles. 1967. Computers in Humanistic Research: Readings and Perspectives. Prentice-Hall.

[9] John Bradley. 2005. What You (Fore)See Is What You Get: Thinking About Usage Paradigms for Computer Assisted Text Analysis. 2 (2005), 20

[10] John Bradley. 2019. Digital Tools in the Humanities: Some Fundamental Provocations? Digital Scholarship in the Humanities 34, 1 (April 2019), 13-20. https://doi.org/10.1093/llc/fqy033

[11] Manuel Burghardt and Christian Wolff. 2015. Humanist-Computer Interaction: Herausforderungen für die Digital Humanities aus Perspektive der Medieninformatik. In DHd Workshop: Informatik und die Digital Humanities. Leipzig.

[12] Manuel Burghardt and Christian Wolff. 2015. Zentren für Digital Humanities in Deutschland. Information - Wissenschaft \& Praxis 66, 5-6 (Nov. 2015). https: //doi.org/10.1515/iwp-2015-0056

[13] Annamaria Carusi and Torsten Reimer. 2010. Virtual Research Environment Collaborative Landscape Study. Report.

[14] Jacob Cohen. 1988. Statistical Power Analysis for the Behavioral Sciences (2nd ed ed.). L. Erlbaum Associates, Hillsdale, N.J.

[15] Jacob Cohen. 1992. A Power Primer. Psychological bulletin 112, 1 (1992), 155.

[16] Fred D. Davis. 1986. A Technology Acceptance Model for Empirically Testing New End-User Information Systems: Theory and Results. Ph.D. Dissertation. Massachusetts Institute of Technology.

[17] Fred D. Davis. 1989. Perceived Usefulness, Perceived Ease of Use, and User Acceptance of Information Technology. MIS Quarterly 13, 3 (Sept. 1989), 319-340. https://doi.org/10.2307/249008

[18] Fred D. Davis. 2006. On the Relationship between HCI and Technology Acceptance Research. In Human-Computer Interaction and Management Information Systems: Foundations, Ping Zhang and Dennis F. Galletta (Eds.). Number vol. 5 in Advances in Management Information Systems. 395-401.

[19] Fred D. Davis, Richard P. Bagozzi, and Paul R. Warshaw. 1989. User Acceptance of Computer Technology: A Comparison of Two Theoretical Models. Management Science 35, 8 (1989), 982-1003.

[20] Adamantios Diamantopoulos, Marko Sarstedt, Christoph Fuchs, Petra Wilczynski, and Sebastian Kaiser. 2012. Guidelines for Choosing between Multi-Item and Single-Item Scales for Construct Measurement: A Predictive Validity Perspective. fournal of the Academy of Marketing Science 40, 3 (May 2012), 434-449. https //doi.org/10.1007/s11747-011-0300-3

[21] Theo K. Dijkstra and Jörg Henseler. 2015. Consistent and Asymptotically Normal PLS Estimators for Linear Structural Equations. Computational Statistics \& Data Analysis 81 (Jan. 2015), 10-23. https://doi.org/10.1016/j.csda.2014.07.008

[22] Theo K. Dijkstra and Jörg Henseler. 2015. Consistent Partial Least Squares Path Modeling. MIS Quarterly 39, 2 (Feb. 2015), 297-316. https://doi.org/10.25300/ MISQ/2015/39.2.02

[23] Aimee L. Drolet and Donald G. Morrison. 2001. Do We Really Need MultipleItem Measures in Service Research? Journal of Service Research 3, 3 (Feb. 2001) 196-204. https://doi.org/10.1177/109467050133001

[24] Yogesh K. Dwivedi, Nripendra P. Rana, Anand Jeyaraj, Marc Clement, and Michael D. Williams. 2019. Re-Examining the Unified Theory of Acceptance and Use of Technology (UTAUT): Towards a Revised Theoretical Model. Information Systems Frontiers 21, 3 (June 2019), 719-734. https://doi.org/10.1007/s10796-017-9774-y

[25] Charlie Edwards. 2012. The Digital Humanities and Its Users. In Debates in the Digital Humanities, Matthew K. Gold (Ed.). Univ Of Minnesota Press, Minneapolis, 213-232.

[26] Martin Fishbein and Icek Ajzen. 1975. Belief, Attitude, Intention, and Behavior: An Introduction to Theory and Research. Addison-Wesley Pub. Co., Reading, Mass.

[27] Claes Fornell and David F Larcker. 1981. Evaluating Structural Equation Models with Unobservable Variables and Measurement Error. JOURNAL OF MARKETING RESEARCH (1981), 12.

[28] Fred Gibbs and Trevor Owens. 2012. Building Better Digital Humanities Tools DH Quarterly 6, 2 (2012).
[29] Lisa M. Given and Rebekah Willson. 2018. Information Technology and the Humanities Scholar: Documenting Digital Research Practices. Fournal of the Association for Information Science and Technology 69, 6 (June 2018), 807-819. https://doi.org/10.1002/asi.24008

[30] David Golumbia. 2013. 'Digital Humanities': Two Definitions.

[31] Dale L. Goodhue, William Lewis, and Ron Thompson. 2012. Does PLS Have Advantages for Small Sample Size or Non-Normal Data? MIS Quarterly 36, 3 (2012), 981. https://doi.org/10.2307/41703490

[32] Dale L. Goodhue and Ronald L. Thompson. 1995. Task-Technology Fit and Individual Performance. MIS Quarterly 19, 2 (June 1995), 213. https://doi.org/10. 2307/249689

[33] Joseph F. Hair (Ed.). 2017. A Primer on Partial Least Squares Structural Equation Modeling (PLS-SEM) (second edition ed.). Sage, Los Angeles.

[34] Joseph F. Hair, Jeffrey J. Risher, Marko Sarstedt, and Christian M. Ringle. 2019. When to Use and How to Report the Results of PLS-SEM. European Business Review 31, 1 (Jan. 2019), 2-24. https://doi.org/10.1108/EBR-11-2018-0203

[35] Patrick Harms and Jens Grabowski. 2011. Usability of Generic Software in EResearch Infrastructures. Fournal of the Chicago Colloquium on Digital Humanities and Computer Science 1, 3 (2011).

[36] Steffen Hennicke, Stefan Gradmann, Kristin Dill, Gerold Tschumpel, Gerold Thoden, Christian Morbindoni, and Alois Pichler. 2015. D3.4 - Research Report on DH Scholarly Primitives. Technical Report. Digitised Manuscripts to Europeana (DM2E).

[37] Jörg Henseler. 2017. Partial Least Squares Path Modeling. In Advanced Methods for Modeling Markets, Peter S. H. Leeflang, Jaap E. Wieringa, Tammo H.A Bijmolt, and Koen H. Pauwels (Eds.). Springer International Publishing, Cham, 361-381. https://doi.org/10.1007/978-3-319-53469-5_12

[38] Jörg Henseler, Theo K. Dijkstra, Marko Sarstedt, Christian M. Ringle, Adamantios Diamantopoulos, Detmar W. Straub, David J. Ketchen, Joseph F. Hair, G. Tomas M. Hult, and Roger J. Calantone. 2014. Common Beliefs and Reality About PLS: Comments on Rönkkö and Evermann (2013). Organizational Research Methods 17, 2 (April 2014), 182-209. https://doi.org/10.1177/1094428114526928

[39] Jörg Henseler, Christian M. Ringle, and Marko Sarstedt. 2015. A New Criterion for Assessing Discriminant Validity in Variance-Based Structural Equation Modeling. Fournal of the Academy of Marketing Science 43, 1 (Jan. 2015), 115-135. https: //doi.org/10.1007/s11747-014-0403-8

[40] Chun Hua Hsiao and Chyan Yang. 2011. The Intellectual Development of the Technology Acceptance Model: A Co-Citation Analysis. International fournal of Information Management 31, 2 (April 2011), 128-136. https://doi.org/10.1016/j. ijinfomgt.2010.07.003

[41] Li-tze Hu, P. M. Bentler, and Yutaka Kano. 1992. Can Test Statistics in Covariance Structure Analysis Be Trusted? Psychological Bulletin 112, 2 (1992), 351-362. https://doi.org/10.1037/0033-2909.112.2.351

[42] K. G. Jöreskog. 1971. Simultaneous Factor Analysis in Several Populations. Psychometrika 36, 4 (Dec. 1971), 409-426. https://doi.org/10.1007/BF02291366

[43] Gohar F. Khan, Marko Sarstedt, Wen-Lung Shiau, Joseph F. Hair, Christian M. Ringle, and Martin P. Fritze. 2019. Methodological Research on Partial Least Squares Structural Equation Modeling (PLS-SEM): An Analysis Based on Social Network Approaches. Internet Research 29, 3 (June 2019), 407-429. https://doi. org/10.1108/IntR-12-2017-0509

[44] Hager Khechine, Sawsen Lakhal, and Paterne Ndjambou. 2016. A Meta-Analysis of the UTAUT Model: Eleven Years Later: A Meta-Analysis of the UTAUT Model: Eleven Years Later. Canadian Journal of Administrative Sciences / Revue Canadienne des Sciences de l'Administration 33, 2 (June 2016), 138-152. https://doi.org/10.1002/cjas.1381

[45] Ned Kock and Pierre Hadaya. 2018. Minimum Sample Size Estimation in PLSSEM: The Inverse Square Root and Gamma-Exponential Methods: Sample Size in PLS-Based SEM. Information Systems fournal 28, 1 (Jan. 2018), 227-261. https: //doi.org/10.1111/isj.12131

[46] Younghwa Lee, Kenneth A. Kozar, and Kai R. T. Larsen. 2003. The Technology Acceptance Model: Past, Present, and Future. In The Influence of Persuasion, Training and Experience on User Perceptions and Acceptance of IT Innovation" Proceedings of the 21 St International Conference on Information Systems. 752-780.

[47] Paul Legris, John Ingham, and Pierre Collerette. 2003. Why Do People Use Information Technology? A Critical Review of the Technology Acceptance Model. Information \& Management 40, 3 (Jan. 2003), 191-204. https://doi.org/10.1016/ S0378-7206(01)00143-4

[48] Gitte Lindgaard, Gary Fernandes, Cathy Dudek, and J. Brown. 2006. Attention Web Designers: You Have 50 Milliseconds to Make a Good First Impression! Behaviour \& Information Technology 25, 2 (March 2006), 115-126. https://doi. org/10.1080/01449290500330448

[49] Qingxiong Ma and Liping Liu. 2004. The Technology Acceptance Model: A Meta-Analysis of Empirical Findings. Fournal of Organizational and End User Computing 16, 1 (Jan. 2004), 59-72. https://doi.org/10.4018/joeuc.2004010104

[50] Nikola Marangunić and Andrina Granić. 2015. Technology Acceptance Model: A Literature Review from 1986 to 2013. Universal Access in the Information Society 14, 1 (March 2015), 81-95. https://doi.org/10.1007/s10209-014-0348-1 
[51] Marcoulides, Chin, and Saunders. 2012. When Imprecise Statistical Statements Become Problematic: A Response to Goodhue, Lewis, and Thompson. MIS Quarterly 36, 3 (2012), 717. https://doi.org/10.2307/41703477

[52] Marcoulides and Saunders. 2006. Editor's Comments: PLS: A Silver Bullet? MIS Quarterly 30, 2 (2006), iii. https://doi.org/10.2307/25148727

[53] Martin Mueller. 2008. Digital Shakespeare, or towards a Literary Informatics. Shakespeare 4, 3 (Sept. 2008), 284-301. https://doi.org/10.1080/17450910802295179

[54] Project Jupyter. 2019. Project Jupyter. https://www.jupyter.org.

[55] Helmut Reinalter and Peter J. Brenner (Eds.). 2011. Lexikon der Geisteswissenschaften: Sachbegriffe - Disziplinen - Personen. Böhlau, Wien.

[56] Oya Y. Rieger. 2010. Framing Digital Humanities: The Role of New Media in Humanities Scholarship. First Monday 15, 10 (Oct. 2010). https://doi.org/10.5210/ fm.v15i10.3198

[57] Edward E. Rigdon. 2012. Rethinking Partial Least Squares Path Modeling: In Praise of Simple Methods. Long Range Planning 45, 5-6 (Oct. 2012), 341-358. https://doi.org/10.1016/j.lrp.2012.09.010

[58] Edward E. Rigdon. 2014. Rethinking Partial Least Squares Path Modeling: Breaking Chains and Forging Ahead. Long Range Planning 47, 3 (June 2014), 161-167. https://doi.org/10.1016/j.lrp.2014.02.003

[59] Edward E. Rigdon. 2016. Choosing PLS Path Modeling as Analytical Method in European Management Research: A Realist Perspective. European Management Journal 34, 6 (Dec. 2016), 598-605. https://doi.org/10.1016/j.emj.2016.05.006

[60] Edward E. Rigdon, Marko Sarstedt, and Christian M. Ringle. 2017. On Comparing Results from CB-SEM and PLS-SEM: Five Perspectives and Five Recommendations. Marketing ZFP 39, 3 (2017), 4-16. https://doi.org/10.15358/0344-1369-20173-4

[61] Ringle, Sarstedt, and Straub. 2012. Editor's Comments: A Critical Look at the Use of PLS-SEM in 'MIS Quarterly'. MIS Quarterly 36, 1 (2012), iii. https: //doi.org/10.2307/41410402

[62] Everett M. Rogers. 1983. Diffusion of Innovations (3rd ed ed.). Free Press ; Collier Macmillan, New York : London.

[63] Jeroen Schepers and Martin Wetzels. 2007. A Meta-Analysis of the Technology Acceptance Model: Investigating Subjective Norm and Moderation Effects. Information \& Management 44, 1 (Jan. 2007), 90-103. https://doi.org/10.1016/j.im. 2006.10.007

[64] Juliane Stiller, Timo Gnadt, Matteo Romanello, and Klaus Thoden. 2016. Anforderungen Ermitteln, Lösungen Evaluieren Und Erfolge Messen - Begleitforschung in DARIAH-DE. Bibliothek Forschung und Praxis 40, 2 (2016), 250-258. https://doi.org/10.1515/bfp-2016-0025

[65] Summit. 2006. Summit on Digital Tools for the Humanities. Technical Report. University of Virginia, Charlottesvill, Virginia.

[66] Patrik Svensson. 2009. Humanities Computing as Digital Humanities. Digital Humanities Quarterly 003, 3 (Sept. 2009).

[67] Hamed Taherdoost. 2018. A Review of Technology Acceptance and Adoption Models and Theories. Procedia Manufacturing 22 (2018), 960-967. https://doi. org/10.1016/j.promfg.2018.03.137

[68] Shirley Taylor and Peter A. Todd. 1995. Understanding Information Technology Usage: A Test of Competing Models. Information Systems Research 6, 2 (June 1995), 144-176. https://doi.org/10.1287/isre.6.2.144

[69] Elaine G. Toms and Heather L. O'Brien. 2008. Understanding the Information and Communication Technology Needs of the E-humanist. Fournal of Documentation 64, 1 (Jan. 2008), 102-130. https://doi.org/10.1108/00220410810844178

[70] Mark Turner, Barbara Kitchenham, Pearl Brereton, Stuart Charters, and David Budgen. 2010. Does the Technology Acceptance Model Predict Actual Use? A Systematic Literature Review. Information and Software Technology 52, 5 (May 2010), 463-479. https://doi.org/10.1016/j.infsof.2009.11.005

[71] Pim van Bree and Geert Kessels. 2013. Nodegoat: A Web-Based Data Management, Network Analysis \& Visualisation Environment. nodegoat.net.

[72] Viswanath Venkatesh and Hillol Bala. 2008. Technology Acceptance Model 3 and a Research Agenda on Interventions. Decision Sciences 39, 2 (May 2008), 273-315. https://doi.org/10.1111/j.1540-5915.2008.00192.x

[73] Viswanath Venkatesh and Fred D. Davis. 2000. A Theoretical Extension of the Technology Acceptance Model: Four Longitudinal Field Studies. Management science 46, 2 (2000), 186-204.

[74] Viswanath Venkatesh, Michael G. Morris, Gordon B. Davis, and Fred D. Davis 2003. User Acceptance of Information Technology: Toward a Unified View. MIS Quarterly 27, 3 (2003), 425-478.

[75] Viswanath Venkatesh, James Thong, Hong Kong University of Science and Technology, Xin $\mathrm{Xu}$, and The Hong Kong Polytechnic University. 2016. Unified Theory of Acceptance and Use of Technology: A Synthesis and the Road Ahead. Journal of the Association for Information Systems 17, 5 (May 2016), 328-376. https://doi.org/10.17705/1jais.00428

[76] Claire Warwick. 2004. Print Scholarship and Digital Resources. In A Companion to Digital Humanities, Susan Schreibman, Raymond George Siemens, and John Unsworth (Eds.). Number 26 in Blackwell Companions to Literature and Culture. Blackwell Pub, Malden, MA.

[77] Claire Warwick. 2012. Studying Users in Digital Humanities. In Digital Humanities in Practice, Claire Warwick, Melissa Terras, and Julianne Nyhan (Eds.). Facet
Publishing, London, 1-21.

[78] Michael D. Williams, Nripendra P. Rana, and Yogesh K. Dwivedi. 2015. The Unified Theory of Acceptance and Use of Technology (UTAUT): A Literature Review. Fournal of Enterprise Information Management 28, 3 (April 2015), 443-488. https://doi.org/10.1108/JEIM-09-2014-0088

[79] Michael D. Williams, Nripendra P. Rana, Yogesh Kumar Dwivedi, and Banita Lal. 2011. Is UTAUT Really Used or Just Cited for the Sake of It? A Systematic Review of Citations of UTAUT's Originating Article. In ECIS.

[80] Shumaila Y. Yousafzai, Gordon R. Foxall, and John G. Pallister. 2007. Technology Acceptance: A Meta-analysis of the TAM: Part 1. fournal of Modelling in Management 2, 3 (Nov. 2007), 251-280. https://doi.org/10.1108/17465660710834453

[81] Shumaila Y Yousafzai, Gordon R Foxall, and John G Pallister. 2007. Technology Acceptance: A Meta-analysis of the TAM: Part 2. (2007), 27.

[82] Ping Zhang and Dennis F. Galletta. 2006. Foundation of Human-Computer Interaction in Management Information Systems. In Human-Computer Interaction and Management Information Systems: Foundations. Number vol. 5 in Advances in Management Information Systems. Routledge, London New York, 1-33. 